\title{
Evaluation of Factors Affecting Mortality in 35 Biliary
}

\section{Tree Injury Cases}

\author{
Tolga Kalayci ${ }^{1 *}$, Ümit Haluk Iliklerden ${ }^{2}$ \\ ${ }^{1}$ Erzurum Regional Education and Research Hospital, Erzurum \\ ${ }^{2}$ Department of General Surgery, V an Yuzuncu Yil University Faculty of Medicine, Van
}

\begin{abstract}
Cholecystectomy is one of the most common operations performed by general surgeons. With the increase in surgeons' skills and advances in technology, laparoscopic cholecystectomy has become the gold standard. However, laparoscopic approach carries a higher risk for biliary tree injury.

In this study, it was aimed to perform clinical analyzes of patients operated on for biliary tree injury and to evaluate the treatment results and mortality factors.

Patients who were operated for bile duct injuries between 1994 and 2018 at Van Yüzüncü Yil University Departmant of General Surgery were selected for the study retrospectively. The data of the patients were collected and the factors determining mortality were evaluated using the Mann-Whitney U test, Chi-Square test and Fisher Exact Test, assuming $\mathrm{p}=$ 0.05 .

Among 35 patients included in this study, 25 were female (71.4\%). The mean age of patients was 45.1 years (22-75). In the etiology, gall bladder operations $(n=29,82.8 \%)$ performed for cholecystolithiasis was the most common. According to the Bismuth-Strasberg classification, while isolated E type injury was seen in 22 patients $(62.9 \%)$, isolated D type injury was seen in 10 patients $(28.6 \%)$. The most performed surgery was hepaticojejunostomy $(48.5 \%)$. Gender, age, first surgery etiology and indication, the day between first surgery and reconstructive surgery, type of injury, type of reconstructive surgery, and hospital stay did not affect mortality ( $\mathrm{p}>0.05)$.

Biliary tree injury is a life-threatening complication of cholecystectomy and should be managed with a multidisciplinary approach. Although no factor affecting mortality was found in this study; late diagnosis and treatment increases both morbidity and mortality.
\end{abstract}

Keywords: Biliary tree injury, Bismuth classification, Hepaticojejunostomy, Laparoscopy, Mortality, Strasberg classification

\section{Introduction}

Cholecystectomy is one of the most common operations performed by general surgeons and gastrointestinal surgeons $(1,2)$. With the increase in surgeons' skills and advances in technology, laparoscopic cholecystectomy has become the gold standard in the treatment of symptomatic cholecystolithiasis. Biliary tree injury is the most common serious and life-threatening complication after cholecystectomy performed for symptomatic cholecystolithiasis.

Despite the advantages of laparoscopic surgery such as short hospital stay, good cosmetic results and rapid recovery, the laparoscopic approach carries a higher risk for iatrogenic biliary tree injury than open surgery. While the rate of iatrogenic injury in laparoscopic surgery is 0.4 $0.6 \%(3,4)$, this rate is $0.125-0.3 \%$ in open surgery $(5,6)$.
Biliary tree injuries occur due to anatomical variations and insufficient experience of the surgeon (2). Some serious complications such as stenosis of the bile ducts or recurrent cholangitis may occur in the long term due to biliary tree injuries. Legal problems arise due to these complications and this issue is a nightmare for surgeons due to the lengthy litigation process.

In this study, we aimed to conduct clinical analyzes of patients who underwent surgery for biliary tree injury and to evaluate the results of treatment in the light of the literature. But mainly, we investigated the factors that affect mortality.

\section{Material and Methods}

Patients who were operated for biliary tree injury between 1994 and 2018 at Van Yüzüncü Yıl University Departmant of General Surgery were selected for the study retrospectively. Hospital records, operation notes, pathology reports, and 
Table 1. Strasberg Classification of Biliary Tree Injuries

\begin{tabular}{lr}
\hline Strasberg classification of biliary tree injuries \\
\hline Type A injury & Leakage into the gallbladder bed from minor hepatic ducts or the cystic duct \\
Type B injury & Injury to aberrant right hepatic ducts due to occlusion \\
Type C injury & Injury to aberrant right hepatic ducts due to transection \\
Type D injury & Lateral damage to the common bile duct \\
Type E injury & Injury to the main ducts; classified according to level of injury \\
(Bismuth Classification) & \\
E1 injury & \\
E2 injury & Injury more than two cm from biliary confluence \\
E3 injury & Injury less than two cm from biliary confluence \\
E4 injury & Injury distal to the confluence, with confluence intact \\
E5 injury & Complete destruction of the biliary confluence \\
\hline
\end{tabular}

clinic charts were evaluated to collect data.

In our hospital electronic archive system has been used since 2010, and patient records before this date have been stored in the archive in written file form. Due to the earthquake that occurred in Van in 2011, the written archive system before 2010 became unusable. We solved this problem as follows. For patients' data between 1994 and 2008, the article published in 2008 by Kotan et al. (7) was used. There were 23 patients included in this study. Unfortunately, data between 2008 and 2010 were not available because of earthquake. The data between 2010 and 2018 was collected from electronic archive. After 2010, it was seen that there were 12 patients eligible for the study. Therefore, pre-2008 and post-2010 data were combined for the study and 35 patients were included in the study.

Patients over 18 years of age and with bile leakage from the liver bed or Luschka duct were excluded from the study. Also, patients who had a preoperative diagnosis of biliary tree injury (BTI) but did not have a BTI injury during surgery were excluded from the study.

Preoperative, intraoperative, and postoperative data of the patients were gathered and factors determining mortality were evaluated. The patients were divided into two groups: the survived $(\mathrm{n}=30)$ and the expired $(n=5)$. The collected parameters were compared with both groups.

\section{Preoperative Factors}

- Demographic features (age and gender)

- The center where the first surgery was performed (If the cases were taken to surgery in an external center, the surgery notes of the surgeon who performed the operation and the notes about the patient were evaluated. The surgeon who performed the operation was discussed about the first operation and the referral indication was learned).

- Indications for the first surgery that caused biliary tree injury

- Time between the first surgery and the reconstructive surgery (secondary surgery, tertiary surgery if present)

- Symptoms and signs in admission

- Diagnostic methods for reconstructive surgery were evaluated as preoperative factors.

\section{Intraoperative Factors}

- Type of surgery performed

- Type of biliary tree injury according to the Bismuth-Strasberg classification (Figure 1 and Table 1) were evaluated as intraoperative factors.

\section{Postoperative Factors}

- Postoperative complications due to BTI, hospital stay, mortality rate were checked for evaluation.

Statistical Analysis: Statistical evaluation was made with SPSS v22.0 (IBM, Armonk, NY, USA). The normality distribution of quantitative variables were checked with Shapiro-Wilk test. The MannWhitney U test was used according to the results of the Shapiro-Wilk test. In addition, Chi-Square test and Fisher Exact Test were used to compare qualitative variables. A p-value below 0.05 was considered statistically significant.

\section{Results}

Among 35 patients included in this study, 25 were female $(71.4 \%)$, and 10 were male $(28.6 \%)$. Female 
Table 2. Preoperative and intraoperative data of patients with distribution of mortality in each factor

\begin{tabular}{|c|c|c|c|}
\hline & $\begin{array}{c}\text { Survived } \\
(\mathrm{n}=30)\end{array}$ & $\begin{array}{c}\text { Expired } \\
(\mathrm{n}=5)\end{array}$ & $\mathrm{p}$ value \\
\hline \multicolumn{4}{|l|}{ Age } \\
\hline$<65$ & $26(86.7 \%)$ & $4(13.3 \%)$ & $0.561 *$ \\
\hline$\geq 65$ & $4(80 \%)$ & $1(20 \%)$ & \\
\hline \multicolumn{4}{|l|}{ Gender } \\
\hline Female & $23(92 \%)$ & $2(8 \%)$ & $0.128 *$ \\
\hline Male & $7(70 \%)$ & $3(30 \%)$ & \\
\hline \multicolumn{4}{|l|}{ Type of first surgery } \\
\hline Open procedures & $13(81.3 \%)$ & $3(18.8 \%)$ & $0.642 *$ \\
\hline Laparoscopic procedures & $17(89.5 \%)$ & $2(10.5 \%)$ & \\
\hline $\begin{array}{l}\text { The day between first surgery and reconstructive } \\
\text { surgery (mean rank) }\end{array}$ & 17.25 & 22.5 & $0.282^{* *}$ \\
\hline \multicolumn{4}{|l|}{ First Operation Center } \\
\hline Internal & $11(91.7 \%)$ & $1(8.3 \%)$ & $0.640 *$ \\
\hline External & $19(82.6 \%)$ & $4(17.4 \%)$ & \\
\hline \multicolumn{4}{|l|}{ Injury Type } \\
\hline Type C & $1(50 \%)$ & $1(50 \%)$ & \\
\hline Type D & $8(80 \%)$ & $2(20 \%)$ & \\
\hline Type E & $19(90.5 \%)$ & $2(9.5 \%)$ & \\
\hline Type E1 & $9(100 \%)$ & $0(0 \%)$ & $0.440 *$ \\
\hline Type E2 & $6(75 \%)$ & $2(25 \%)$ & \\
\hline Type E3 & $4(100 \%)$ & $0(0 \%)$ & \\
\hline Combine Type A + Type D & $1(100 \%)$ & $0(0 \%)$ & \\
\hline Combine Type E1 + Type E3 & $1(100 \%)$ & $0(0 \%)$ & \\
\hline Type of Surgery & $15(88.2 \%)$ & $2(11.8 \%)$ & \\
\hline Hepaticojejunostomy & $10(76.9 \%)$ & $3(23.1 \%)$ & \\
\hline T-tube drainage with or without primary repair & \\
\hline Choledocoduodenostomy & $2(100 \%)$ & $0(0 \%)$ & $0.849^{*}$ \\
\hline Whipple procedure & $1(100 \%)$ & $0(0 \%)$ & \\
\hline Primary repair & $1(100 \%)$ & $0(0 \%)$ & \\
\hline Choledocojejunostomy & $1(100 \%)$ & $0(0 \%)$ & \\
\hline Hospital Stay (mean rank) & 17.33 & 22 & $0.345^{* *}$ \\
\hline
\end{tabular}

*Chi-Square Test results, ** Mann-Whitney U Test results

to male ratio was 2.5 . While the mean age of men was 45.1 (25-71) years, the mean age of women was $45.1(22-75) .85 .7 \%(n=30)$ of all cases were below 65 years. Both gender and age (being over 65 year) did not affect mortality, $\mathrm{p}=0.128$ and $\mathrm{p}=0.561$, respectively. Preoperative and intraoperative parameters are shown in Table 2.

In the etiology of BTI, gall bladder operations $(\mathrm{n}=29,82.8 \%)$ performed for cholecystolithiasis was the most common. 19 of these surgeries were performed laparoscopically and eight were open. In two gall bladder surgeries, the operations were started laparoscopically. However, due to difficulty in dissection, open surgery was performed. There was no correlation between mortality and first surgery type $(p=0.642)$. The first surgical indications that cause BTI and the centers where the operations are performed were shown in Figure 2.

23 patients $(65.7 \%)$ with BTI were referred from secondary health centers. BTI had occurred in 21 of these patients during the gallbladder operation. BTI due to gallbladder operation occurred in 14 patients during laparoscopic surgery and in two patients during open surgery. BTI, which was seen outside the gallbladder operation, occurred during 


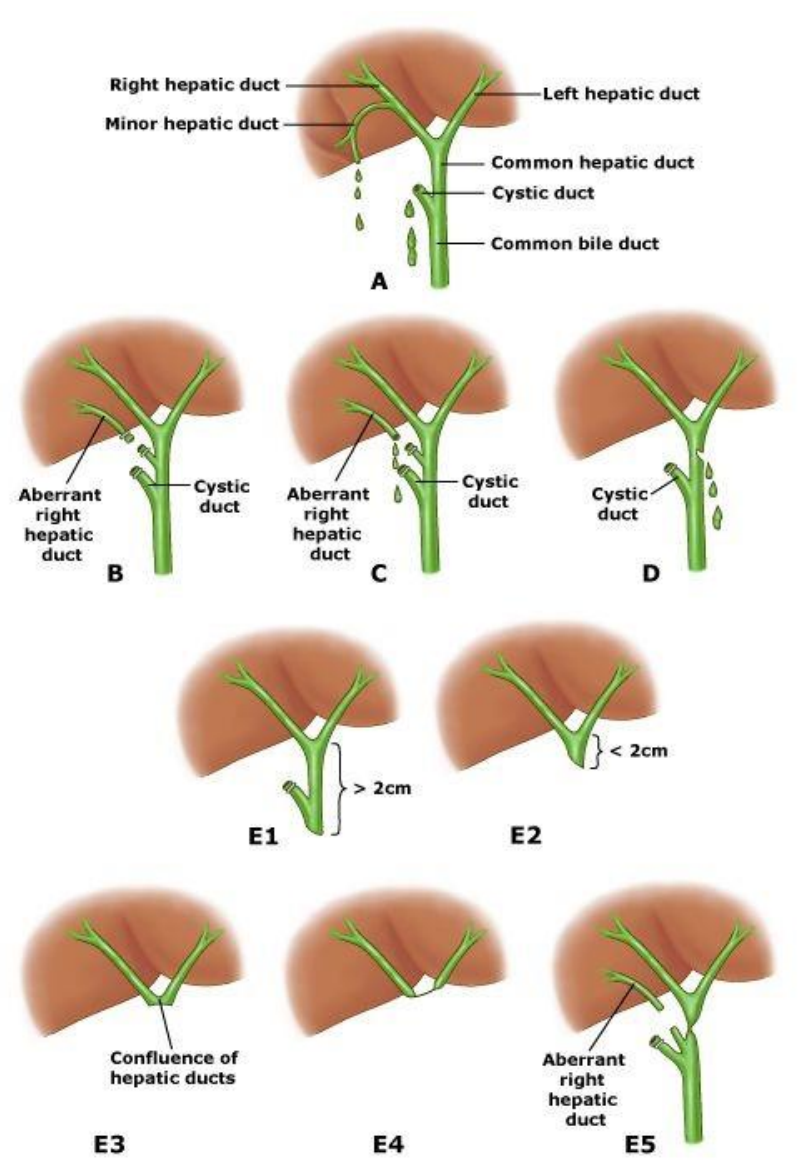

Fig. 1. Bismuth-Strasberg classification of biliary tree injuries

operations performed for trauma and acute abdomen. There were 12 cases of BTI seen in our clinic. The most common surgical indication of BTI was cholecystolithiasis ( $n=8, \quad 66.8 \%)$. Operation indications of the remaining four cases were common bile duct injury during Percutaneous Transhepatic Cholangiography, injury during recurrent peptic ulcer disease surgery, gastric outlet obstruction and liver hydatid cyst, respectively.

Peritonitis-related abdominal pain and jaundice were the most common symptoms at presentation. However, abdominal distension, fever, and sepsis were other less common symptoms. The laboratory changes and ultrasonography results of all patients could not be evaluated due to lack of data before 2008. However, according to post2010 data, six of 12 patients had an increase in leukocyte count, eight had an increase in bilirubin (both total and direct), and 5 had an increase in Creactive protein. In addition, massive intraabdominal fluid was detected in the ultrasonography before reconstructive surgery in nine patients.

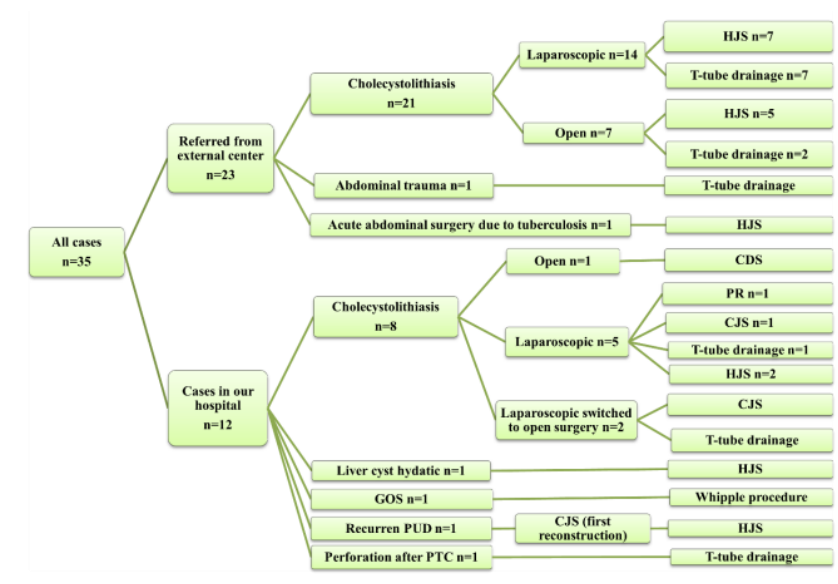

Fig. 2. Treatment algorithm of patients. HJS: Hepaticojejunostomy. CJS: Choledocojejunostomy. GOO: Gastric Outlet Obstruction. PUD: Peptic Ulcer Disease. CDS: Choledocojejunostomy. PR: Primary Repair

MRCP (magnetic resonance cholangiopancreatography) was used to diagnose BTI in 14 of 35 patients. While 12 of these patients had leakage on MRCP report, two patients had no leakage.

Endoscopic intervention was not performed on any patient before reconstructive surgery, and all patients were treated surgically. While in 10 patients, reconstructive surgery for BTI was performed at the same session, other patients were operated on different day. The average time between the first surgery and the operation due to injury was 13.5 days (range 3 to 59 days).

According to the Bismuth-Strasberg classification, while isolated $E$ type injury was seen in 22 patients $(62.9 \%)$, isolated D type injury was seen in 10 patients $(28.6 \%)$. The number of cases according to injury types is shown in Table 2 . The injury presented in two patients was a combination of two types of injuries. One of the combinations was Type A injury and Type D injury combination, the other combination was Type E1 injury and Type E3 injury combination. There was no difference in mortality between type E injury and type D injury $(p=0.572)$

The most performed surgery was hepaticojejunostomy (48.5\%). The types of surgery applied to the patients are shown in Figure 2. In one patient reconstructive surgery was performed twice. While choledocojejunostomy was performed as the first reconstructive surgery, hepaticojejunostomy was performed as the second reconstructive surgery due to bile leakage. There was no difference in mortality between hepaticojejunostomy and T-tube drainage $(p=0.628)$. 
The mean hospital stay was 21.8 days (0-67 days). Mortality rate was $14.2 \% \quad(n=5)$. There were no factors affecting mortality in this study (Table 2). The average follow-up period of patients 33.2 months (range from 2 months to 147 months). In postoperative follow-up, only one patient had biliary stricture. And biliary stricture was treated with endobiliary stent.

\section{Discussion}

Cholecystolithiasis mainly occurs as an asymptomatic disease and is discovered incidentally during imaging methods requested for the diagnosis of other abdominal diseases. Only about $20 \%$ of patients with asymptomatic cholecystolithiasis becomes symptomatic. Nowadays laparoscopic cholecystectomy has become preferred surgery in the treatment of symptomatic cholecystolithiasis. Although the recovery process in the postoperative period of patients who have undergone laparoscopic surgery is fast, it increases the risk of biliary tree injury (8). Biliary tree injury (BTI) occurs most often after cholecystectomy. Also, BTI is the most feared complication with high morbidity $(9.3 \%-43 \%)$ and early mortality $(0 \%-1.7 \%)(8,9)$. BTI can also occur after trauma especially penetrating, liver cyst hydatic, gastric outlet obstruction, or other invasive procedures like Percutaneous Transhepatic Cholangiography (PTC), or Endoscopic Retrograde Cholangiopancreatography (ERCP). Regardless of the etiology, legal problems caused by BTI can turn into nightmares for surgeons. Thus, the diagnosis and treatment process of BTI should be known by surgeons. In this study, $82.8 \%$ of all patients operated because of cholecystolithiasis (acute or chronic). The remaining surgeries were performed due to acute abdomen, trauma, common bile duct injury during percutaneous transhepatic cholangiography, injury during recurrent peptic ulcer disease surgery, gastric outlet obstruction and liver hydatid cyst.

In BTI, there is no definite superiority according to gender. While female gender was dominant in most studies $(9,10)$, male gender was found dominant in the study of Acar et al. (11). In this study, the female gender was the dominant gender with $71.4 \%$. As in the study (45.1 years), the average age in the literature ranged from 45 to 55 years $(9-12)$.

Early detection of BTI is an important issue both morbidity and mortality (12,13). Clinical symptoms depend on type of injury, extent of injury and leak diagnosis time. As the diagnosis is delayed, the amount of biloma collected intraabdominal cavity increases. As the amount of fluid increases, the severity of peritonitis increases. In early period, if the drain inserted into the abdomen during the first surgery is still present, the amount of bile coming from the drain is diagnostic. As time goes by, patients begin to develop symptoms of peritonitis such as abdominal distention, jaundice, fever, and cholangitis.

The most common symptoms in this study were peritonitis-related abdominal pain and jaundice due to peritonitis. While BTI of 10 patients was noticed in the same operation, BTI was noticed in 25 patients in the postoperative period. The earliest BTI was detected on the $3^{\text {rd }}$ postoperative day. Therefore, most patients had peritonitis due to late diagnosis.

Laboratory and radiological investigations play important role in the diagnosis of BTI. In laboratory, infective parameters like leukocyte and C-reactive protein levels increase depending on the severity of peritonitis. Jaundice occurs when bile flow is obstructed, or bile passes into the peritoneal cavity. Since there is no damage in liver cells, there is no increase in aminotransferase levels, but an increase in cholestatic enzymes such as alkaline phosphatase and $\gamma$-glutamyl transpeptidase. In the study of Kotan et al. (7), there was no data on laboratory changes. However, according to post-2010 data, six of 12 patients had an increase in leukocyte count, eight had an increase in bilirubin (both total and direct), and five had an increase in $\mathrm{C}$-reactive protein.

Ultrasonography (USG) is first choice in diagnose of BTI. USG can provide information about intrahepatic and extrahepatic bile ducts. USG can also show biloma in patients with bile leakage. Computed tomography is useful for more specific examination in patients with suspected bile leakage. Percutaneous transhepatic cholangiography is useful in evaluating the proximal biliary tree at the location of the injury. ERCP is an extremely useful research method for imaging damaged bile ducts and allows repair of small bile duct injuries by inserting a bile stent. Magnetic resonance cholangiography is a sensitive (85\%-100\%) and non-invasive imaging method for the bile ducts. It is currently the "gold standard" in the preoperative diagnosis of BTI in patients eligible for surgical reconstruction (12-14). According to the post-2010 data, USG was used in nine of 12 patients to evaluate BTI, and USG showed massive fluid (possibly biloma), 
disseminated into the abdomen. MRCP was used to diagnose BTI in 14 patients in recent study and sensitivity of MRCP for BTI was $85.7 \%$.

\section{ERCP (Endoscopic Retrograde} Cholangiopancreaticography) was first treatment method for BTI. In the ERCP procedure, a catheter or plastic stent (in various sizes) is placed after sphincterotomy according to the imaging results. Depending on the type of injury, ERCP has a success rate of up to $100 \%$ in the literature (15-17).

Surgical management remains gold standard for BTI if injury cannot be managed with ERCP. There are several approaches to repair BTI. Surgical methods include ligation or primary repair of duct, end-to-end anastomosis of interrupted bile duct, biliary enteric anastomosis, transplantation and partial hepatectomy $(18,19)$. While the preferred operative repair depends on the injury pattern, Roux-en-Y Hepaticojejunostomy is the most common procedure in the surgical treatment of BTI, as in this study $(20,21)$. If there is no evidence of common bile duct and extensive peritonitis in patients, the results are more satisfying. However, there are studies reporting that the leakage area will expand due to the tension in the anastomosis line and may cause leakage. On the other hand, up to $80 \%$ stenosis may develop in long-term followup (22). Stenosis occurred in only one patient and the stenosis was successfully treated with a stent.

In our hospital, electronic archive system has been used since 2010, and patient records before this date have been stored in the archive in written file form. Due to the earthquake that occurred in Van in 2011, the written archive system before 2010 became unusable. For patients' data between 1994 and 2008, the article published in 2008 by Kotan et al. (7) was used. Unfortunately, data between 2008 and 2010 were not available because of earthquake. The data between 2010 and 2018 was collected from electronic archive. This is the most important limitation of this study.

BTI is a life-threatening complication of cholecystectomy and should be managed with a multidisciplinary approach. The process should be managed in cooperation with the radiologist, gastroenterologist and experienced surgeons. Surgeons with little or no experience with BTI should refer cases to the nearest experienced center as early as possible.

Another important issue is to detect BTI early before peritonitis develops. Late diagnosis and treatment complicates the management of cases and increases both morbidity and mortality. Early treatment prevents serious complications. Patients require long-term and careful follow-up due to the risk of stricture after bile duct repair.

Conflict of Interest: No conflict of interest was declared by the authors.

Financial Disclosure: No financial disclosure was declared by the authors.

\section{References}

1. Gupta V, Gupta A, Yadav TD, Mittal BR, Kochhar R. Post-cholecystectomy acute injury: What can go wrong? Ann Hepatobiliary Pancreat Surg 2019; 23: 138-144.

2. Chuang KI, Corley D, Postlethwaite DA, Merchant M, Harris HW. Does increased experience with laparoscopic cholecystectomy yield more complex bile duct injuries? Am J Surg 2012; 203: 480-487.

3. Martin D, Uldry E, Demartines N, Halkic N. Bile duct injuries after laparoscopic cholecystectomy: 11-year experience in a tertiary center. Biosci Trends 2016; 10: 197-201.

4. Mishra PK, Saluja SS, Nayeem M, Sharma BC, Patil N. Bile duct injury-from injury to repair: An analysis of management and outcome. Indian J Surg 2015; 77: 536-542.

5. Wysocki AP. Population-based studies should not be used to justify a policy of routine cholangiography to prevent major bile duct injury during laparoscopic cholecystectomy. World J Surg 2017; 41:82-89.

6. Tantia O, Jain M, Khanna S, Sen B. Iatrogenic biliary injury: 13.305 cholecystectomies experienced by a single surgical team over more than 13 years. Surg Endosc 2008; 22: 1077-1086.

7. Kotan C, Kisli E, Lobut N, Kiziltan R, Uslukaya $\mathrm{O}$, Bartin $\mathrm{K}$ et al. Outcomes of the surgical treatment of thr bile duct injuries: Evaluation of 23 cases. Journal of Turk HPB 2008; 4: 99-105.

8. Berney CR. Major common bile duct injury and risk of litigation: a surgeon's perspective. Am J Surg 2012; 204: 800-802.

9. Maddah G, Rajabi Mashhadi MT, Parvizi Mashhadi M, Nooghabi MJ, Hassanpour M, Abdollahi A. Iatrogenic injuries of the extrahepatic biliary system. J Surg Res 2017; 213: 215-221.

10. Slater K, Strong RW, Wall DR, Lynch SV. Iatrogenic bile duct injury: The scourge of laparoscopic cholecystectomy. ANZ Journal of Surgery 2002; 72: 83-88.

11. Acar T, Acar N, Güngör F, Alper E, Gür Ö, Çamyar $\mathrm{H}$, et al. Endoscopic and surgical management of iatrogenic biliary tract injuries. Ulus Travma Acil Cerrahi Derg 2020; 26: 203 211. 
12. Barbier L, Souche R, Slim K, Ah-Soune P. Longterm consequences of bile duct injury after cholecystectomy. J Visc Surg 2014; 151: 269-279.

13. Rystedt J, Lindell G, Montgomery A. Bile duct injuries associated with 55.134 cholecystectomies: Treatment and outcome from a National Perspective. World J Surg 2016; 40: 73-80.

14. Pesce A, Palmucci S, La Greca G, Puleo S. Iatrogenic bile duct injury: impact and management challenges. Clin Exp Gastroenterol 2019; 12: 121-128.

15. Rainio M, Lindstrom O, Udd M, Haapamaki C, Nordin A, Kylanpaa L. Endoscopic therapy of biliary injury after cholecystectomy. Dig Dis Sci 2018; 63: 474-480.

16. Dumonceau JM, Tringali A, Blero D, Deviere J, Laugiers R, Heresbach D, et al. Biliary stenting: indications, choice of stents and results European Society of Gastrointestinal Endoscopy (ESGE) clinical guideline. Endoscopy 2012; 44: 277-298.

17. Kim KH, Kim TN. Endoscopic management of bile leakage after cholecystectomy: a single-center experience for 12 years. Clin Endosc 2014; 47: 248-253.
18. Pekolj J, Alvarez FA, Palavecino M, Claria RS, Mazza O, Santibanes E. Intraoperative management and repair of bile duct injuries sustained during 10.123 laparoscopic cholecystectomies in a high-volume referral center. J Am Coll Surg 2013; 216: 894-901.

19. Bharathy KGS, Negi SS. Postcholecystectomy bile duct injury and its sequelae: pathogenesis, classification, and management. Indian J Gastroenterol 2013; 33: 201-215.

20. Viste A, Horn A, Ovrebo K, Christensen B, Angelsen JH, Hoem D. Bile duct injuries following laparoscopic cholecystectomy. Scand J Surg 2015; 104: 233-237.

21. Shetty S, Desai PR, Vora HB, Bhavsar MS, Khiria LS, Yadav A et al. Management of major postcholecystectomy biliary injuries: An analysis of surgical results in 62 patients. Niger J Surg 2019; 25: 91-96.

22. Stewart L. Iatrogenic biliary injuries: identification, classification, and management. Surg Clin North Am 2014; 94: 297-310. 